\title{
REFLECTION OF WAVES FROM VARYING MEDIA*
}

\author{
BY \\ C. O. HINES** \\ Radio Physics Laboratory, Defence Research Board, Ottawa
}

\begin{abstract}
Formulae are found for the coefficient of reflection from varying media of a type encountered in physics. These are applied approximately for some general classes of media, and exactly for some specific cases. Many media which would normally be expected to be highly reflecting are shown to be completely transparent to certain waves at least and, in some cases, to a whole spectrum of waves. The results are considered both for electromagnetic (or other classical) waves and for mass waves.

I. Introduction and Summary. Many problems of wave propagation through varying media introduce the equation

$$
\frac{d^{2} f}{d x^{2}}+g(x) f=0
$$

for solution. This equation was studied some years ago by Lord Rayleigh [1] and others in connection with classical wave problems. The advent of quantum mechanics has led to a more thorough investigation of the solutions of (1), centered around the method developed by Brillouin [2], Wentzel [3] and Kramers [4] - the B.W.K. method. More recent work along these lines has been carried out by Kemble [5, 6], Langer [7, 8, 9], and Furry [10]. Exact solutions, in terms of hypergeometric functions, can be found if $g(x)$ is of the proper form; this method of attack was developed by Eckart [11] and Epstein [12]. In all these treatments, great attention has been paid to solutions obtained when $g(x)$ is positive for some ranges of $x$ and negative for others. Such solutions are of particular interest in quantum mechanics, in some oblique incidence problems, and in problems of electromagnetic wave propagation through a conducting medium such as the ionosphere.

In the present paper we will deal with functions $g(x)$ which are everywhere real, finite, and continuous, have a constant positive value for large values of $|x|$, and lesser values in a region of variation about $x=0$.

Solutions are obtained in terms of a new variable $r=r(x)$ which is itself a solution of a Riccati equation. $\dagger$ Exact formulae, also in terms of $r$, are developed for the reflection coefficient of a medium. The curve of $r(x)$ is traced by inspection of the determining differential equation. A surprisingly large amount of information about $r$ can be found in this way, but the procedure is only qualitative.

By this method, then, we obtain an approximate evaluation of the exact solution, rather than an exact evaluation of an approximate solution as is found in the B.W.K. method. This difference of approach leads to a difference in applicability. The B.W.K. method is of prime importance in deriving numerical results in a particular given problem, although it has been used to infer some general results. The present method

*Received Sept. 12, 1951. Received in revised form March 14, 1952.

**Present address: Fitzwilliam House, Cambridge, England.

†See the paper by S. A. Schelkunoff [16] for comments on the occurrence of the Riccati equation in wave transmission problems and, indeed, for a number of interesting and pertinent remarks on the whole problem of propagation under varying conditions. 
leads to the construction of problems to which it can give an exact solution, predicts some general results, and indicates some surprising (?) results which can be obtained if the medium is of the proper form. In particular, it is shown that many media which might be expected, by inference, to be highly reflecting can be completely transparent to one frequency (or energy) at least. This is particularly true of media in which $g(x)$ is negative in two separated regions; in this case a whole spectrum may be transmitted.

The formulae are developed in the symbolism commonly used in electromagnetic wave propagation, but the results are also considered in terms of mass waves.

II Electromagnetic Waves. In the electromagnetic wave problem, an equation of the form of (1) comes from eliminating time, $t$, from

$$
\frac{\partial^{2} \Psi}{\partial x^{2}}-\frac{n^{2}}{c^{2}} \frac{\partial^{2} \Psi}{\partial t^{2}}=0
$$

by setting

$$
\Psi=f(x) \epsilon^{-i k c t} .
$$

One thus obtains

$$
\frac{d^{2} f}{d x^{2}}+k^{2} n^{2} f=0
$$

Here $n^{2}=n^{2}(x)$ is the effective permittivity, $c$ is the vacuum speed of light, and $k$ is the (real positive) circular wave number in vacuum.

We shall here be concerned only with media for which $n^{2}=n^{2}(x)$ is real, finite, continuous, and such that

$$
\begin{array}{lll}
n=1 & \text { for } & |x|>x_{a}>0 \\
n^{2} \leq 1 & \text { for } & |x|<x_{a} .
\end{array}
$$

These conditions are imposed primarily for convenience of discussion, and most may be removed if proper modifications in the development are made.

Introduce $r=r(x)$ by defining

$$
f=\exp \left[i k \int_{x_{0}}^{x} r(s) d s\right]
$$

where $x_{0}$ is an arbitrary real constant. Substituting this into (4) we get

$$
n^{2}=r^{2}-\frac{i}{k} \frac{d r}{d x}
$$

as the equation determining $r(x)$. We shall allow only continuous solutions of (7) so that $f$ and $d f / d x$ will be continuous. Three solutions of (7) will be of particular interest to us; they will be denoted $r^{+}, r^{-}$, and $r^{\prime}$, and are characterised by the following conditions:

$$
r^{+}=1 \quad \text { for } \quad x \leq-x_{a},
$$




$$
\begin{array}{lll}
r^{-}=-1 & \text { for } & x \leq-x_{a}, \\
r^{\prime}=1 & \text { for } & x \geq x_{a} .
\end{array}
$$

Writing

$$
r^{+}=r_{1}+i r_{2}
$$

where $r_{1}$ and $r_{2}$ are real, equation (7) yields

$$
\begin{gathered}
n^{2}=r_{1}^{2}-r_{2}^{2}+\frac{1}{k} \frac{d r_{2}}{d x}, \\
0=2 r_{1} r_{2}-\frac{1}{k} \frac{d r_{1}}{d x},
\end{gathered}
$$

since we are taking $n^{2}$ to be real. Equation (13) gives immediately

$$
r_{2}=\frac{1}{2 k} \frac{d}{d x} \log \left|r_{1}\right|
$$

by which we obtain

$$
\int_{x_{0}}^{x} r^{+}(s) d s=\int_{x_{0}}^{x} r_{1}(s) d s+\frac{i}{2 k} \log \left|\frac{r_{1}(x)}{r_{1}\left(x_{0}\right)}\right| .
$$

For convenience, take $x_{0}=-x_{a}$ so that, by (8) and (11)

$$
r_{1}\left(x_{0}\right)=r_{1}\left(-x_{a}\right)=1 \text {, }
$$

and hence we can write

$$
f^{+}=\frac{1}{\sqrt{\left|r_{1}(x)\right|}} \exp \left[i k \int_{-x_{\bullet}}^{x} r_{1}(s) d s\right]
$$

as a solution of (4). It will be shown in Part IV that $r_{1}(x)$ is always positive, hence the absolute value bars may be dropped.

The expression

$$
F^{+} f^{+} \epsilon^{-i k c t}=\frac{F^{+}}{\sqrt{r_{1}(x)}} \exp \left[i k \int_{-x_{\bullet}}^{x} r_{1}(s) d s-i k c t\right]
$$

with $\mathrm{F}^{+}$an arbitrary complex constant, will then be a solution of (2). In the region to the left of the variation (i.e. for $x<-x_{a}$, by convention) this represents a wave of amplitude $F^{+}$and speed $c$ moving to the right. In the region to the right of the variation $\left(x>x_{a}\right)$, it must represent, in general, two waves, one moving to the right and the other to the left, each with speed $c$.

It can be seen by inspecting (12) and (14) that if $r_{1}+i r_{2}$ is a solution of (7), so too is $-r_{1}+i r_{2}$; this is, in fact, simply $r^{-}$. Hence we have a second solution of (2) namely

$$
F^{-} f^{-} \epsilon^{-i k c t} \equiv \frac{F^{-}}{\sqrt{r_{1}(x)}} \exp \left[i k \int_{-x_{0}}^{x} r_{1}(s) d s-i k c t\right] .
$$

Remarks similar to those above apply to this solution also. 
The two solutions obtained are independent, the Wronskian of the system $f^{+}, f^{-}$ having the constant value $-2 i k$, hence the general solution of $(2)$ is

$$
\Psi=\left(F^{+} f^{+}+F^{-} f^{-}\right) \epsilon^{-i k c t}
$$

with $F^{+}$and $F^{-}$arbitrary complex constants.

Another solution of (2) is

$$
F^{\prime} f^{\prime} \epsilon^{-i k c t} \equiv \frac{F^{\prime}}{\sqrt{r_{1}^{\prime}(x)}} \exp \left[i k \int_{x_{a}}^{x} r_{1}^{\prime}(s) d s-i k c t\right] .
$$

Here we have taken $x_{0}=x_{a}$ and $r^{\prime}=r_{1}^{\prime}+i r_{2}^{\prime}$, with $r_{1}^{\prime}$ and $r_{2}^{\prime}$ real. This solution represents, in the region to the right of the variation, a single wave moving to the right.

III. Formulae for Reflection Coefficients. In obtaining reflection coefficients, we consider solutions of (2) which give a single (transmitted) wave leaving the region on one side or the other. If this occurs on the left we must have $F^{+}=0$, and the complete solution is then given by (19). If the single wave moves off on the right, then the ratio $F^{-} / F^{+}$must be such that the left-moving components on the right cancel out. In this case, however, we can equally well give the complete solution by (21). For equivalence,

$$
F^{+} f^{+}+F^{-} f^{-}=F^{\prime} f^{\prime}
$$

and, differentiating this with respect to $x$ and dividing out $i k$,

$$
r^{+} F^{+} f^{+}+r^{-} F^{-} f^{-}=r^{\prime} F^{\prime} f^{\prime} .
$$

From these we obtain the complex amplitude reflection coefficient

$$
\begin{aligned}
R & \equiv \frac{F^{-}}{F^{+}}=-\frac{r^{+}-r^{\prime}}{r^{-}-r^{\prime}} \frac{f^{+}}{f^{-}} \\
& =-\frac{\left(r_{1}-r_{1}^{\prime}\right)+i\left(r_{2}-r_{2}^{\prime}\right)}{\left(-r_{1}-r_{1}^{\prime}\right)+i\left(r_{2}-r_{2}^{\prime}\right)} \exp \left[2 i k \int_{-x_{*}}^{x} r_{1}(s) d s\right]
\end{aligned}
$$

which has modulus

$$
|R|=\left|\frac{\left(r_{1}-r_{1}^{\prime}\right)+i\left(r_{2}-r_{2}^{\prime}\right)}{\left(r_{1}+r_{1}^{\prime}\right)+i\left(r_{2}-r_{2}^{\prime}\right)}\right| .
$$

Since $R$ is a constant, the evaluation may be made at any $x$.

If the variation is symmetrical about $x=0$-i.e.,if $n^{2}(x)=n^{2}(-x)$-then it may easily be shown that

$$
r_{1}^{\prime}(x)=r_{1}(-x) ; \quad r_{2}^{\prime}(x)=-r_{2}(-x) .
$$

In particular, at $x=0$ we get $r_{1}^{\prime}=r_{1}$ and $r_{2}^{\prime}=-r_{2}$. Substituting this in (25), we get

$$
\left|R_{s}\right|=\left[1+r_{1}^{2}(0) / r_{2}^{2}(0)\right]^{-1 / 2}
$$

for a symmetrical medium.

It may be noted that formulae (22) to (25) would be valid even if $n^{2}$ were complex in the region $|x|<x_{a}$ 
IV. Approximate Curves for $\mathbf{r}^{+}(\mathbf{x})$. No general solution of the Riccati equation is known, so we are apparently no further ahead. However, the sort of solution to be expected can be estimated, to a certain extent, by inspection of the equations (12) to (14), and this task will be undertaken in the present part. The development is necessarily lengthy, and has only been outlined here. However qualitative it may be, it does lead to some general results and does provide a guide for further extension, as illustrated in succeeding parts. Moreover, since the required curves are shown to be smooth, at least over the regions of interest, it appears that electronic computers might be used to advantage in this sort of approach to the problem. (Contrast the actual wave solution which is, in the main, oscillatory.) The operator $D$ will be used for $d / d x$ throughout the discussion.

We shall be concerned here primarily with fairly slowly varying media, having simple curves of $n^{2}(x)$, in which $n^{2}$ is negative over some range, say $x_{b}<x<x_{d}$. A representation of such a medium is shown in Fig. 1. Media in which $n^{2}$ is slowly varying but never becomes negative will be classified as Type I, and will receive only scant treatment, near the end.

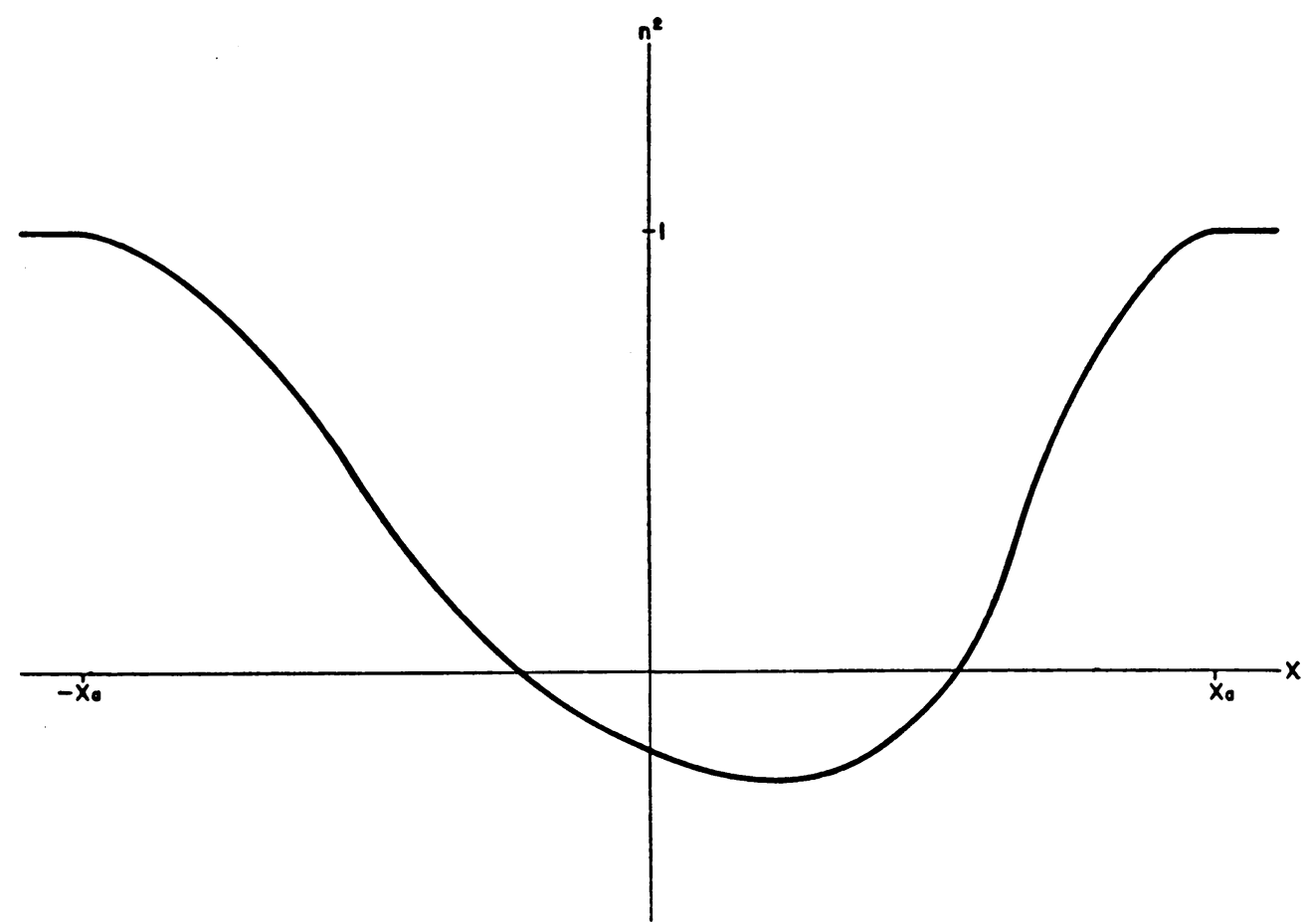

Fig. I TYPICAL MEDIUM TO BE CONSIDERED

We start from the region $x<-x_{a}$, where $r_{1}=1$ and $r_{2}=0$, and proceed to the right. Since $r^{+}$is continuous, no change can occur in either $r_{1}$ or $r_{2}$ until one occurs in $D r_{2}$. Since $n^{2}$ starts to decrease, we see from (12) that $D r_{2}$ must become negative. Then $D^{2} r_{1}$ and $D r_{1}$ become negative, and $r_{1}^{2}$ decreases from unity. So long as $3\left(D r_{1}\right)^{2}>$ $2 r_{1} D^{2} r_{1}$, however, $r_{1}^{2}$ must stay greater than $n^{2}$, as may be seen on eliminating $r_{2}$ from (12) and (14). This gives, for the start of the $r_{1}^{2}$ and $r_{2}\left|r_{2}\right|$ curves the form shown in 
Fig. 2. (We plot $r_{2}\left|r_{2}\right|$ so that the magnitude of $r_{2}^{2}$ and the signs of $r_{2}$ and $D r_{2}$ may be shown.)

Before proceeding farther, we might first note two general properties of the $r_{1}^{2}$ curves, based on (12) and (14):

i) $r_{1}$ will never become zero (if $x_{a}$ is finite) for this would require $r_{2} \rightarrow-\infty$, and hence $n^{2} \rightarrow-\infty$, a case which has been excluded from the present paper.

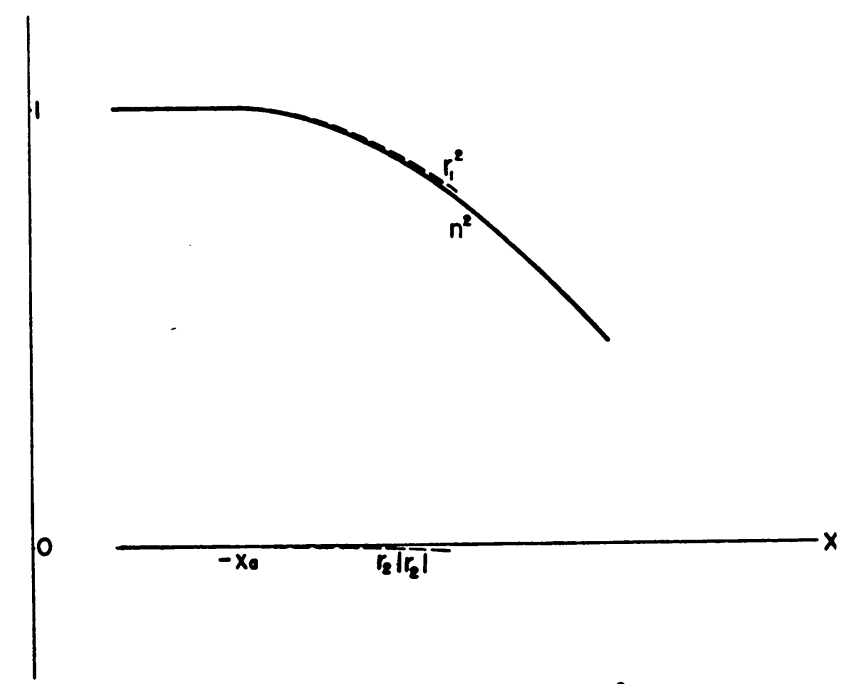

Fig. 2 STARTING CURVES OF $r_{1}^{2}$ AND $r_{2}\left|r_{2}\right|$

ii) if ever $D r_{1}^{2}$ becomes zero after having been negative, so too will $D r_{1}$, hence at that point $r_{2}=0$ and $D r_{2}>0$, and so $n^{2}>r_{1}^{2}$. Hence, so long as $r_{1}^{2}$ remains greater than $n^{2}$, it will not have a zero derivative.

For slowly varying media we may expect slowly varying $r$ curves, in which case we will have $r_{1}^{2} \approx n^{2}$ until very close to $x_{b}$, where $n^{2}$ vanishes. Such a result is, in fact,

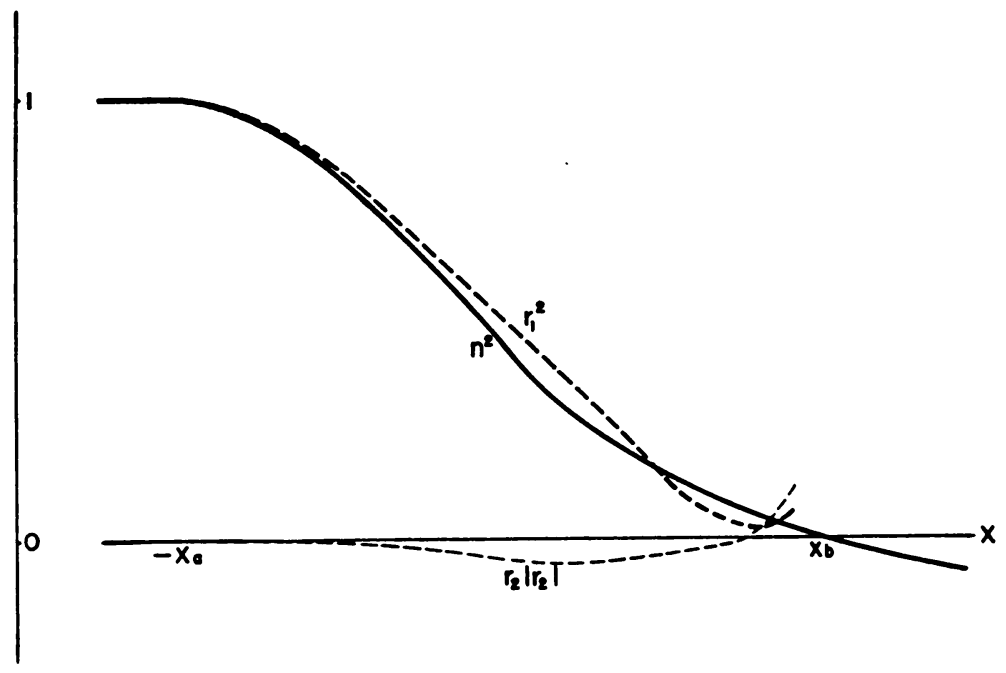

Fig. 3 POSSIBLE LATER CURVES $r_{1}^{2}$ AND $r_{2}\left|r_{2}\right|$ 
employed in the B.W.K. method where, in effect, $r_{1} \approx n$ (for $x<x_{b}$ ) is derived from the first term of a series solution of (12) and (14), and then employed in (18). Except in the limit of vanishing variation per wave length $(k \rightarrow \infty)$ we cannot know that this result must be obtained, but the reverse certainly is true: if $r_{1}$ and $D r_{1}$ are very slowly varying, then $n^{2}$ is too, and $r_{1}^{2} \approx n^{2}$. Later curves of $r_{1}^{2}$ and $r_{2}\left|r_{2}\right|$ may then be like those in Fig. 3 or, more likely if the medium is slowly varying, like those in Fig. 4 or 5.

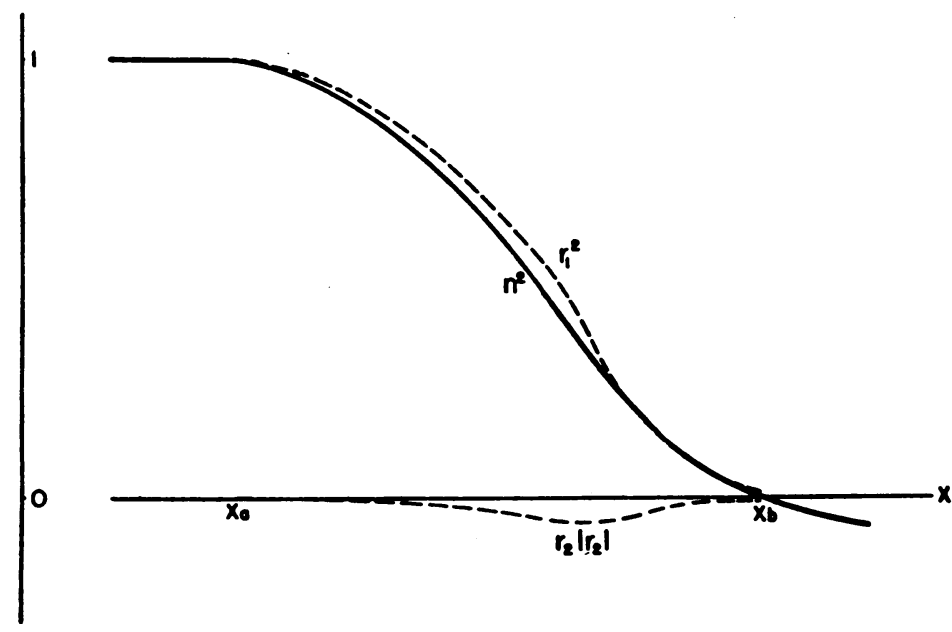

Fig. 4 POSSIBLE LATER CURVES OF $r_{1}^{2}$ AND $r_{2}\left|r_{2}\right|$

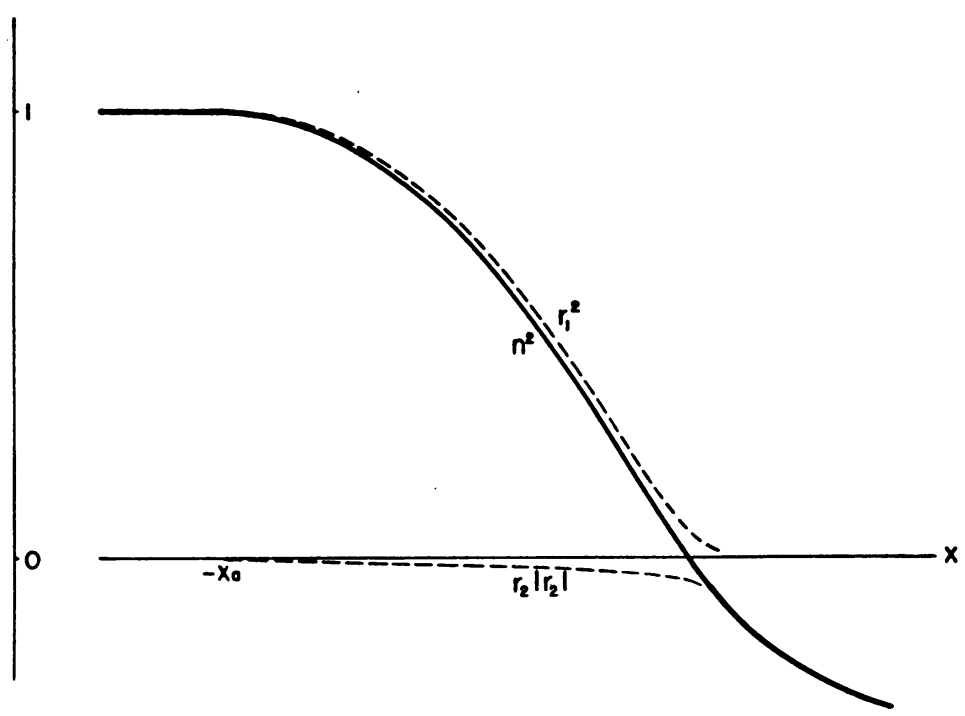

Fig. 5 POSSIBLE LATER CURVES OF $r_{1}^{2}$ AND $r_{2}\left|r_{2}\right|$

At any rate, when we come to $x_{b}$ we have $n^{2}=0, r_{1}^{2}>0$. Media having $D r_{1}>0$ at $x_{b}$ will be classified as Type II while those having $D r_{1}<0$ there will be classified as Type III. There does not appear to be any general way of continuing the curves past 
$x_{b}$ for media of Type II, which we shall therefore drop from our discussion for the present. Type III can, however, be treated generally, as follows:

As before, there will be no point in the region $x_{b}<x<x_{d}$ where $D r_{1}=0=r_{2}$, since the property (ii) above applies to Type III media there. This means, then, that $D r_{1}<0$ and $r_{2}<0$ throughout this region. Moreover, if the medium is sufficiently slowly varying, $r_{1}^{2}$ will not differ from $n^{2}$ by very much in the region to the left of $x_{b}$, and hence will be very small at, and to the right of, $x_{b}$. In fact, it can be shown that

$$
\int_{x_{b}}^{x_{d}} r_{1} d x<\pi / 2 k
$$

for any medium of Type III. (This is easily deduced from the fact that the graph of the real part of $f$ must be curved away from the $x$-axis in regions of negative $n^{2}$.) From this we see that $r_{1}$ must become quite small between $x_{b}$ and $x_{d}$ if $k\left(x_{d}-x_{b}\right)$ is reasonably large, no matter how large $r_{1}$ is at $x_{b}$.

Proceeding to the right, we see that if $-r_{2}^{2}$ is less than $n^{2}$ by more than the (small) amount $r_{1}^{2}$, then $D r_{1}$ must be positive and $-r_{2}^{2}$ must be increasing. So long as $n^{2}$ is not increasing, then, $-r_{2}^{2}$ cannot be much less than $n^{2}$ for long. Once $-r_{2}^{2}$ becomes greater than $n^{2}-r_{1}^{2}$ it must remain there, at least until this latter starts to increase (near $x_{m}$, the point of minimum $n^{2}$ ). All that time $D r_{2}$ will be negative, and so $-r_{2}^{2}$ will be decreasing. If the gap between $-r_{2}^{2}$ and $n^{2}-r_{1}^{2}$ becomes large, so too will $-D r_{2}$, thus forcing the gap to diminish (assuming fairly slow variation of $n^{2}$ ). Figs. 6 and 7 show the type of curves to be expected.

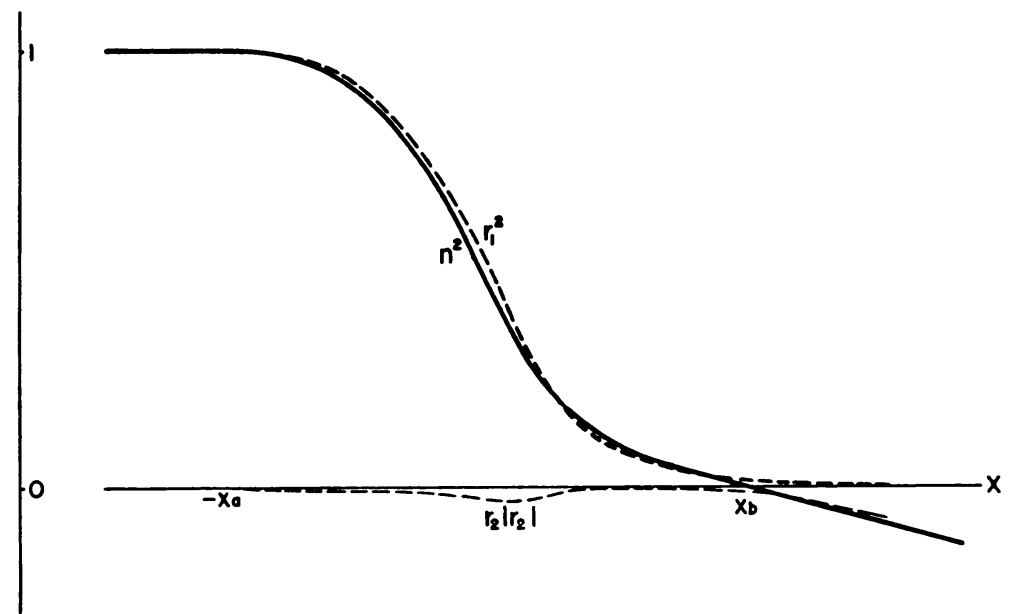

Fig. 6 POSSIBLE CURVES OF $r_{1}^{2}$ AND $r_{2}\left|r_{2}\right|$, SHOWING $-r_{2}^{2}$ BECOMING GREATER THAN $n^{2}$

All these considerations hold especially well when the variation is sufficiently slow. In the limiting case $k \rightarrow \infty$ we will have $r_{1} \rightarrow n, r_{2} \rightarrow 0$ for $x<x_{b}$, and $r_{1} \rightarrow 0, r_{2} \rightarrow$ $-|n|$ for $x_{b}<x<x_{d}$. As we relax this condition of slow variation, the considerations above are modified. Inspection shows that this leads to curves such as those sketched in Figs. 8, 9, 10, and 11. They are, of course, only rough drawings meant to indicate the sort of curves to be expected; they are not mathematically accurate, and "slow variation" is not defined.

In most cases of slow variation, the curves can be extended as far to the right as 


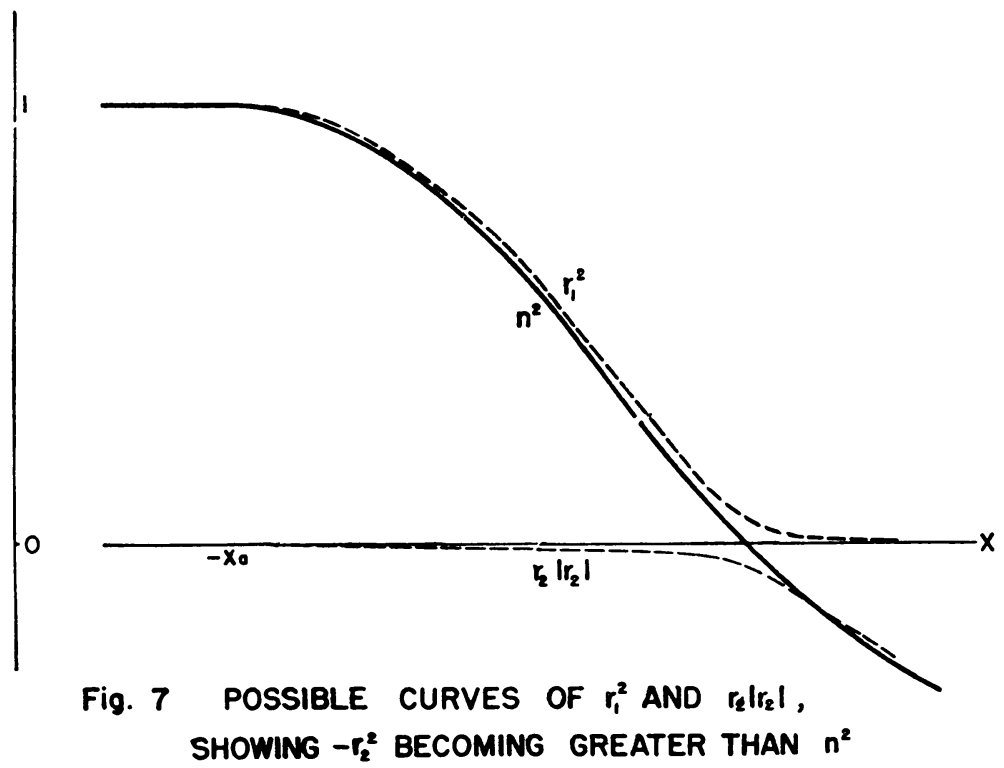

$x_{d}$ without too much error. After this point, however, it is impossible to continue by inspection, for $r_{1}$ and its derivatives become large. However, we do know that

$$
\exp \left[i k \int_{-x_{\bullet}}^{x} r^{+}(s) d s\right]=A \epsilon^{i k n x}+B \epsilon^{-i k n x}
$$

for $x>x_{a}$. Here $A$ and $B$ are complex constants and, in our case, $n=1$. From this can be derived

$$
r^{+}(x)=n \frac{A \epsilon^{i k n x}-B \epsilon^{-i k n x}}{A \epsilon^{i k n x}+B \epsilon^{-i k n x}} .
$$

In general (i.e. $B \neq 0$ ) this gives oscillatory curves for $r_{1}$ and $r_{2}$.

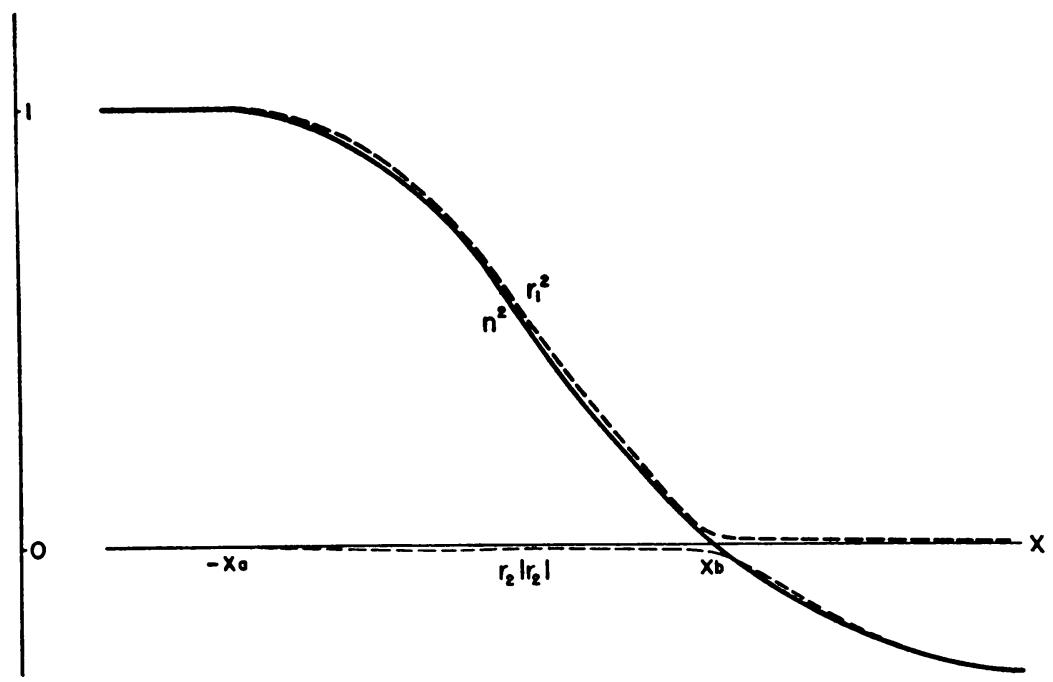

Fig. $8 r_{1}^{2}$ AND $r_{2}\left|r_{2}\right|$ CURVES FOR A VERY SLOWLY VARYING MEDIUM OF TYPE III 

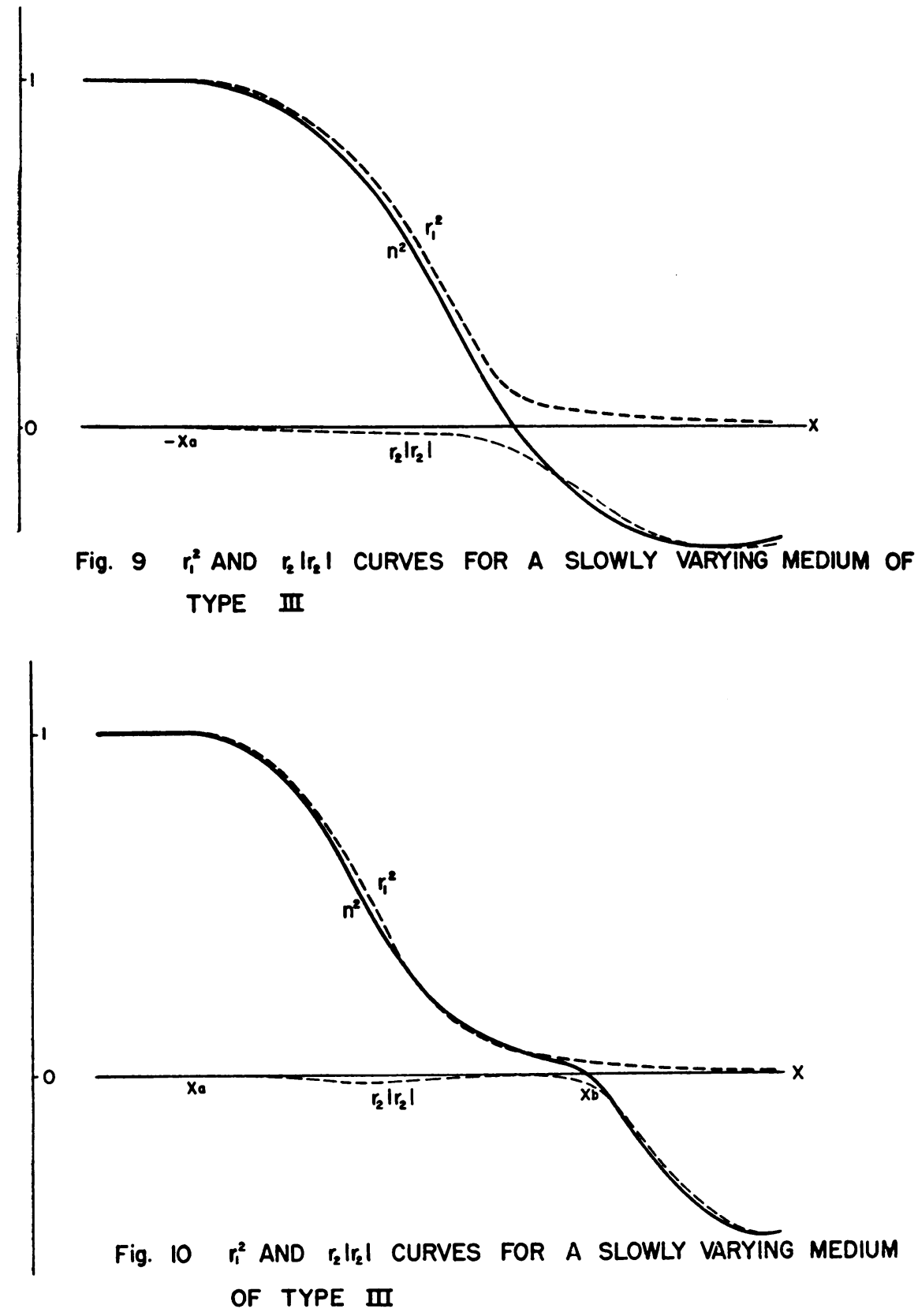

Actually, there is no need to describe the curves past $x_{m}$, since the formulae (25) and (27) can be used at $x_{m}$ itself. We can trace the curves for $r_{1}^{\prime 2}$ and $r_{2}^{\prime}\left|r_{2}^{\prime}\right|$, working to the left of $x_{a}$ in a similar way, to the region of the minimum of $n^{2}$. This gives a complete set of curves for the medium, such as that shown for a typical case in Fig. 12. Other media will have curves differing in detail from these, but we may take it as a fairly general result that media having a single fairly wide region of negative $n^{2}$ will have $r_{1}$ and $r_{1}^{\prime}$ quite small, and $-r_{2}$ and $r_{2}^{\prime}$ approximately equal to $|n|$, at $x_{m}$. 


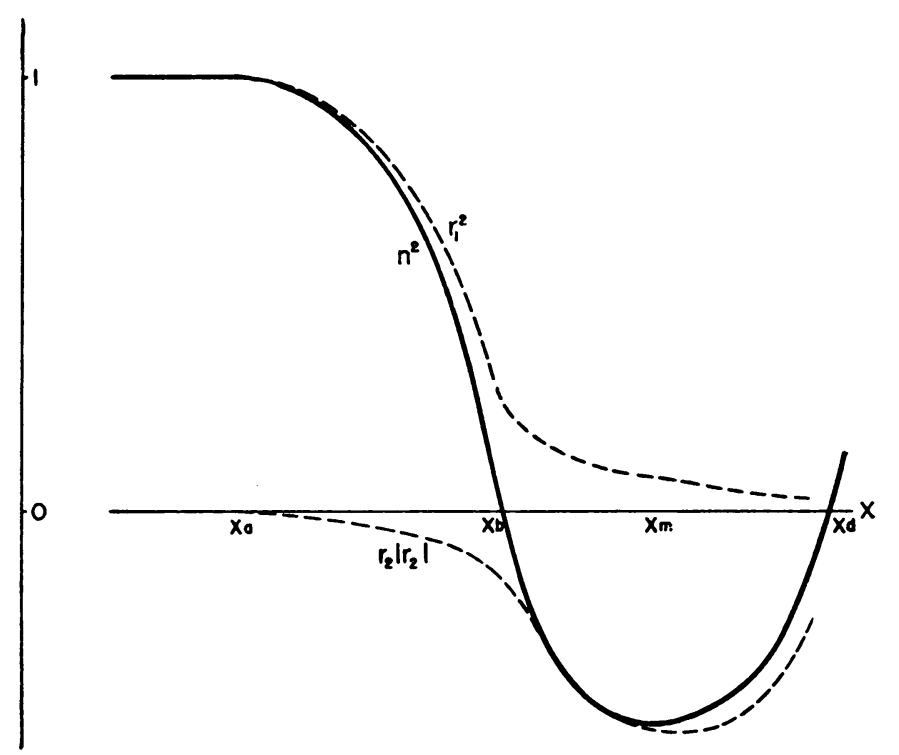

Fig. II $r_{1}^{2}$ AND $r_{2}\left|r_{2}\right|$ CURVES FOR A FAIRLY RAPIDLY VARYING MEDIUM OF TYPE III

This last statement will be true even for media of Type II, since it can be shown for them that

$$
\int_{x_{b}}^{x_{d}} r_{1} d x<\pi / k
$$

If, then, $n^{2}$ stays negative for long enough, $r_{1}$ must become very small. Along with

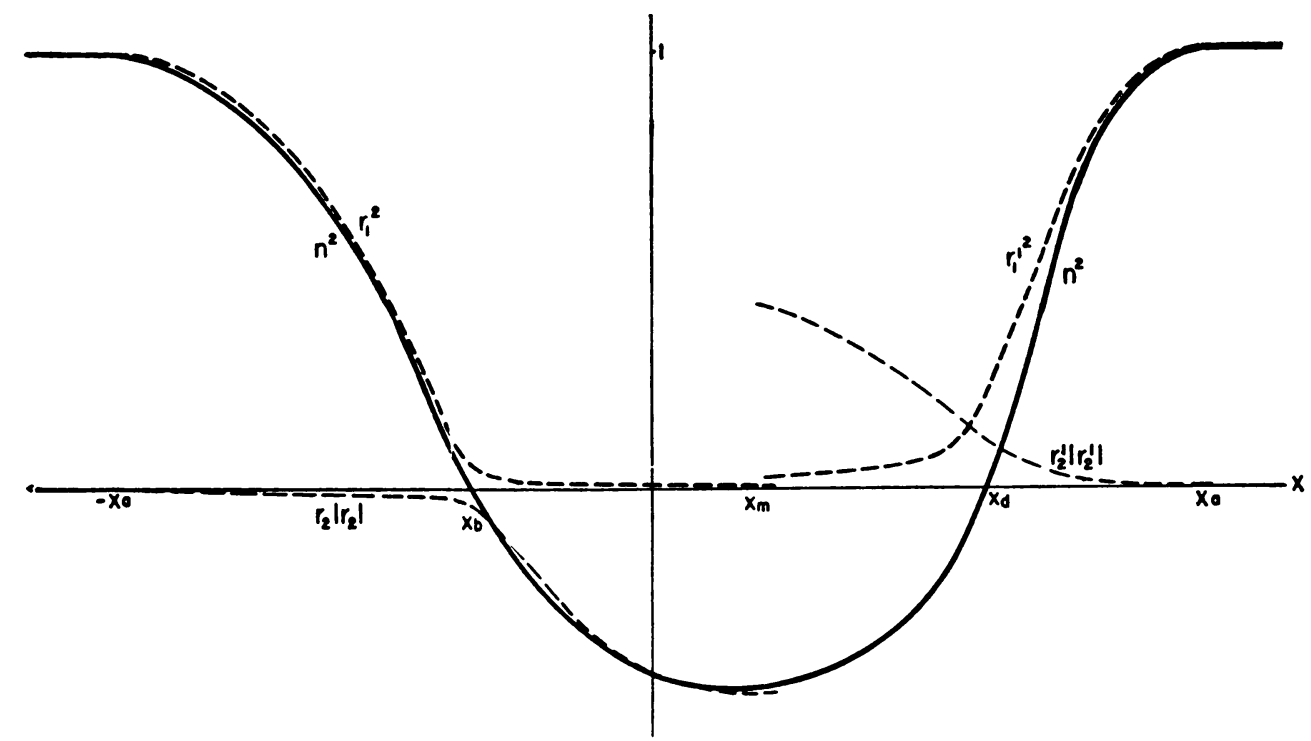

Fig. 12 CURVES OF $r_{1}^{2}, r_{2}\left|r_{2}\right|, r_{1}^{2}$, AND $r_{2}^{\prime}\left|r_{2}^{\prime}\right|$ FOR A FAIRLY SLOWLY VARYING MEDIUM OF TYPE III ON BOTH SIDES 
this, $D r_{1}$ must become negative, and the results derived above for Type III media will become applicable.

In Type I media having $n^{2}$ slowly varying, we expect the approximation $r_{1} \approx n$, $r_{2} \approx 0$, to be fairly good throughout the entire range, at least if the minimum $n^{2}$ is not too close to zero.

V. Approximate Reflection Coefficients. i) Type I media, minimum $n^{2}>0$ : So long as the approximations $r_{1} \approx n \approx r_{1}^{\prime}, r_{2} \approx 0 \approx r_{2}^{\prime}$ are good, (25) gives $|R| \approx 0$. If these approximations break down, as will happen in rapidly varying media, the resulting reflection coefficient may become appreciable.

ii) Type I media, minimum $n^{2}=0$ : It can be seen that, under certain circumstances (e.g. $r_{1} \approx-r_{2}, r_{1}^{\prime} \approx r_{2}^{\prime}, D r_{2} \approx 0 \approx D r_{2}^{\prime}$ at $x_{m}$ ) we will obtain $|R| \approx 1 \sqrt{2}$. This is a result frequently quoted for this case (e.g. Rydbeck [13]), but as can be seen, it is by no means general. An example will be given (Part IX, Fig. 20) in which it completely breaks down.

iii) Media having a broad region of negative $n^{2}$ : We will have, at $x_{m},-r_{2} \gg r_{1}>0$, $r_{2}^{\prime} \gg r_{1}^{\prime}>0$, and hence $|R| \approx 1$. We thus get the expected result, that such media are almost completely reflecting.

iv) Symmetrical media: On integrating (14) and introducing the result into (27) we obtain for symmetrical media

$$
\left|R_{s}\right|=\left[1+\frac{1}{r_{2}^{2}(0)} \exp \left\{4 k \int_{-x_{a}}^{0} r_{2} d x\right\}\right]^{-1 / 2} .
$$

This gives a form which may be compared with the common (approximate) formula

$$
|R| \approx\left[1+\exp \left\{-2 k \int_{x_{b}}^{x_{d}}|n| d x\right\}\right]^{-1 / 2}
$$

which becomes, for symmetrical media

$$
\left|R_{s}\right| \approx\left[1+\exp \left\{-4 k \int_{x_{b}}^{0}|n| d x\right\}\right]^{-1 / 2} .
$$

This was originally developed by Gamow [14] by considering discontinuous media, and more recently by the "good path" method [6] [13]. If $n^{2}$ is sufficiently negative for a sufficient range of $x$, both methods give $\left|R_{s}\right| \approx 1$, as in (iii). However, for rapidly varying media which are not too thick, (32) [or in general, (25)] and (33) may give quite different results.

v) Thin media of Type II: The reflection coefficients for such media may differ considerably from the "good path" result, and so somewhat surprising values may occur. An example is given in Part IX, Fig. 21.

VI. Accurate Reflection Coefficients. Regardless of the accuracy or inaccuracy of the discussion in Part IV when applied to general cases, the formulae (25) and (27) can be used for specially constructed media as follows:

Use any function $\rho_{1}(x)$ which equals 1 for $x<-x_{a}$, and remains positive and has a continuous first derivative everywhere. Define

$$
\rho_{2}(x)=\frac{1}{2 k} \frac{d}{d x} \log \rho_{1}
$$


and construct a medium for which

$$
n^{2}=\rho_{1}^{2}-\rho_{2}^{2}+\frac{1}{k} \frac{d}{d x} \rho_{2}
$$

using any specified $k$.

For this medium, at the frequency $k c / 2 \pi$, we will have

$$
r_{1}=\rho_{1} ; \quad r_{2}=\rho_{2}
$$

exactly. To satisfy the conditions (5) on $n, \rho_{1}$ would have to have a certain form for $x>x_{a}$. This difficulty can be avoided, however, by using another function $\rho_{1}^{\prime}$ which equals 1 for $x>x_{a}$, and remains positive and has a continuous first derivative everywhere. The medium to be used will have $n^{2}$ given by (35) for $x<x_{c}$ and by a similar relation in $\rho_{1}^{\prime}, \rho_{2}^{\prime}$ for $x>x_{e}, x_{e}$ being a point where the two relations give the same value. (The existence of at least one such point is a further condition, though we may now remove the restrictions on $\rho_{1}$ to the right, and those on $\rho_{1}^{\prime}$ to the left, of $x_{e}$. )

We should then be able to derive the exact reflection coefficient for the medium so constructed, at the frequency $k c / 2 \pi$. Using the discussion of Part IV as a guide, one could obtain a specified form of medium. For media with large regions of negative $n^{2}$, it is easier to choose $\rho_{1}$ and $\rho_{1}^{\prime}$ over part of the range only, and choose $\rho_{2}$ and $\rho_{2}^{\prime}$ over the remainder $\left(x_{b}<x<x_{d}\right)$. Examples are given in Part IX.

It should be pointed out that this technique is accurate in all cases. The variations may be as rapid as we please, and $n^{2}$ need not be restricted by the conditions imposed in this paper (see, for example, Fig. 26, Part IX, where $n \neq 1$ on the right). Even complex $n^{2}$ 's may be obtained, if proper modifications are made. There are certain disadvantages to the method, however:

i) The reflection coefficient is only known for the frequency used in the construction. For nearby frequencies we may expect little change, but we have no way of estimating the amount. In some cases (see Part VIII) the frequency is very critical in determining reflection. However, if we choose functions to give curves as in Fig. 12, we can be fairly sure that for higher frequencies the curves approach the limiting values mentioned before (aside from dispersion effects).

ii) The exact form of the medium, i.e. $n^{2}=n^{2}(x)$, is not known until after the construction is made. However, with proper judgment, we could construct almost any medium desired, at least approximately.

iii) In general, $d n^{2}: d x$ will be discontinuous at $x=x_{e}$. This is not a serious fault, however, and need not occur if care is taken in selecting the $\rho$ functions.

VII. Non-Reflecting Media. One of the primary values of the method just developed is that it shows the existence of non-reflecting media (for any given frequency) even when $n^{2}$ is considerably negative over a considerable distance. To see this, we note that if $\rho_{1}$ is taken so it equals 1 for $x>x_{a}$, then we may take $\rho_{1}^{\prime}=\rho_{1}$, hence $r_{1}=r_{1}^{\prime}$, $r_{2}=r_{2}^{\prime}$, and hence $|R|=0$. Using the great freedom of choice of $\rho_{1}$ allowed, we can construct a great variety of media for which the reflection coefficient is zero (for the frequency used in construction). This applies to rapidly varying media as well as slowly varying ones. The possibility of non-reflecting media was noted by Rayleigh [1], based on similar reasoning, but not developed by him nor applied for cases of negative $n^{2}$.

If we choose $\rho_{1}$ so that $\rho_{2}$ is appreciable when $\rho_{1} \approx 0$ we can get a medium with $n^{2}$ appreciably negative (and for as great a distance as we wish) which has the property 
of non-reflection (for the frequency used). Several non-reflecting media are sketched in Figs. 13, 14, 15, and 16, the last two being of this type.

All non-reflecting media of the types considered in this paper must have

$$
\int_{-x_{a}}^{x_{a}} r_{2}(x) d x=0
$$

in order that $r_{1}\left(x_{a}\right)=r_{1}\left(-x_{a}\right)$. Since regions of negative $n^{2}$ usually arise due to $\left|r_{2}\right|$ becoming large, we can expect that a non-reflecting medium having one region of negative $n^{2}$ (due, say, to $-r_{2}$ becoming large) will have another one (due to $+r_{2}$ becoming

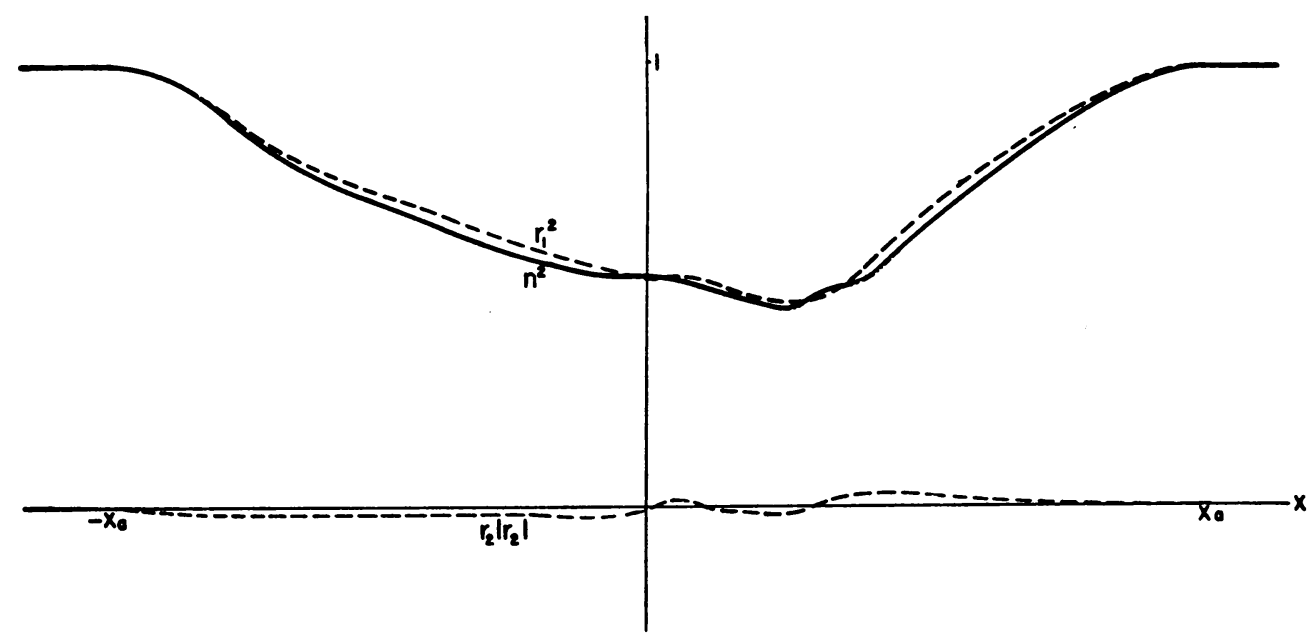

Fig. I3 A NON-REFLECTING MEDIUM (FOR A CERTAIN FREQUENCY). $r_{1}^{2}$ CURVE PRESCRIBED

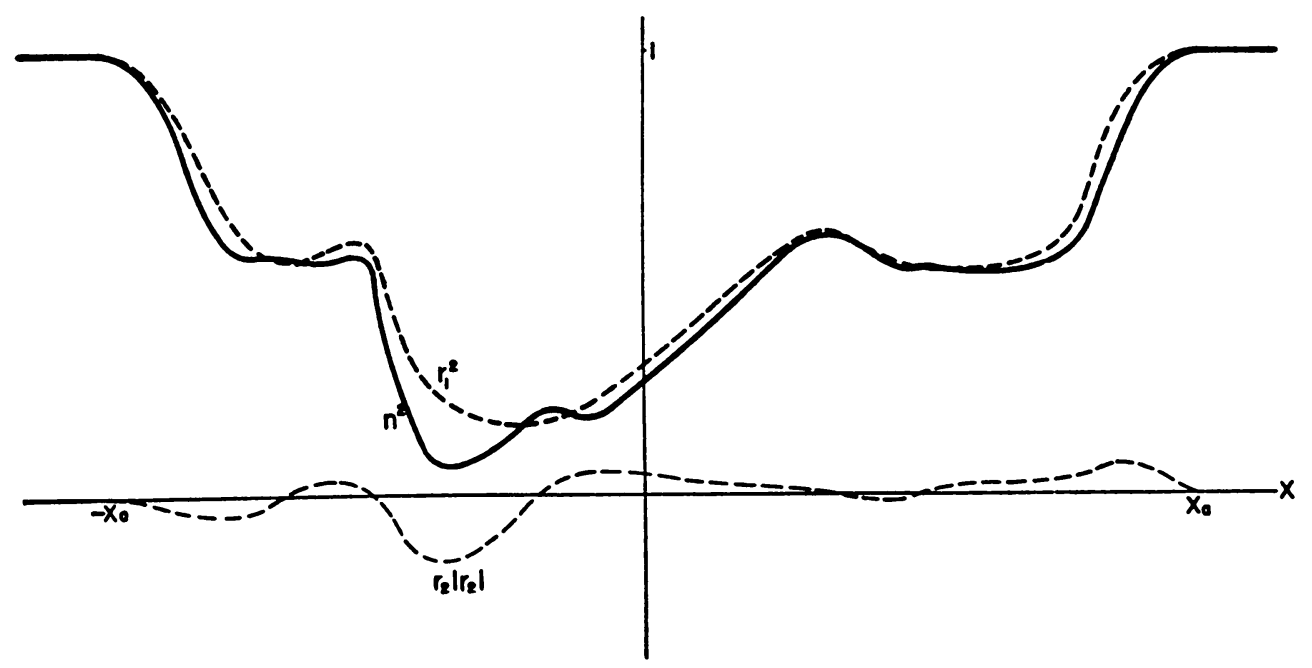

Fig. 14 A NON-REFLECTING MEDIUM (FOR A CERTAN FREQUENCY). $r_{1}^{2}$ CURVE PRESCRIBED 


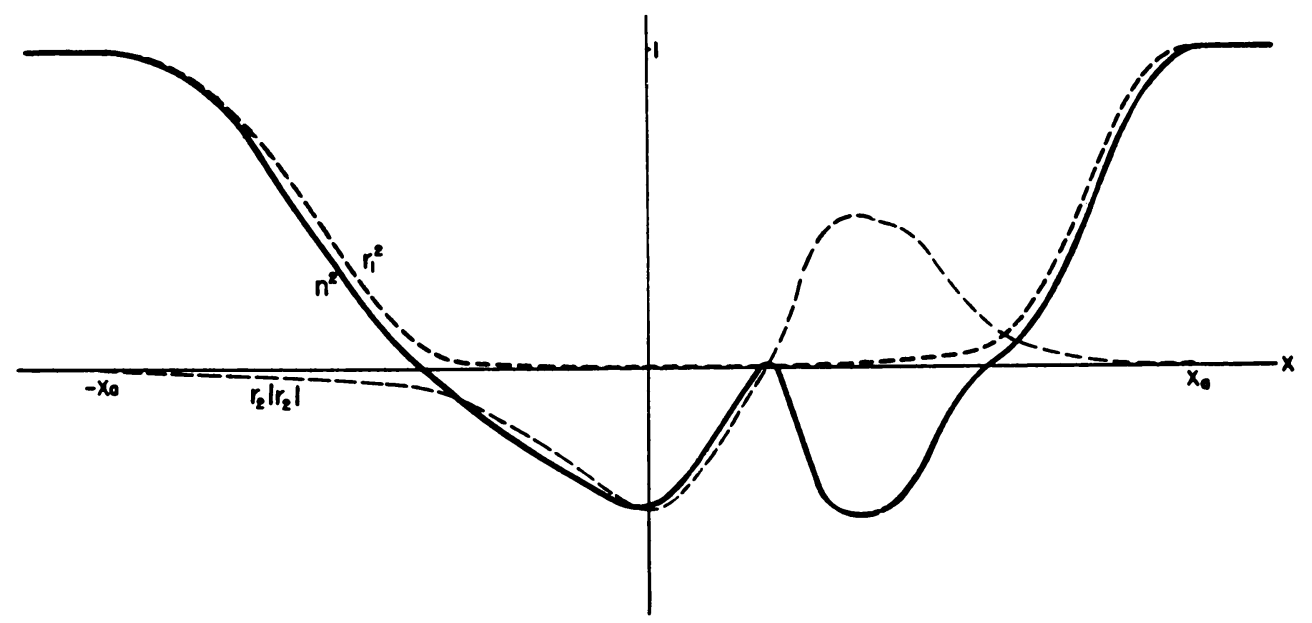

Fig. I5 A NON-REFLECTING MEDIUM (FOR A CERTAIN FREQUENCY) WITH TWO REGIONS OF NEGATIVE $n^{2}$. $r_{1}^{2}$ CURVE PRESCRIBED

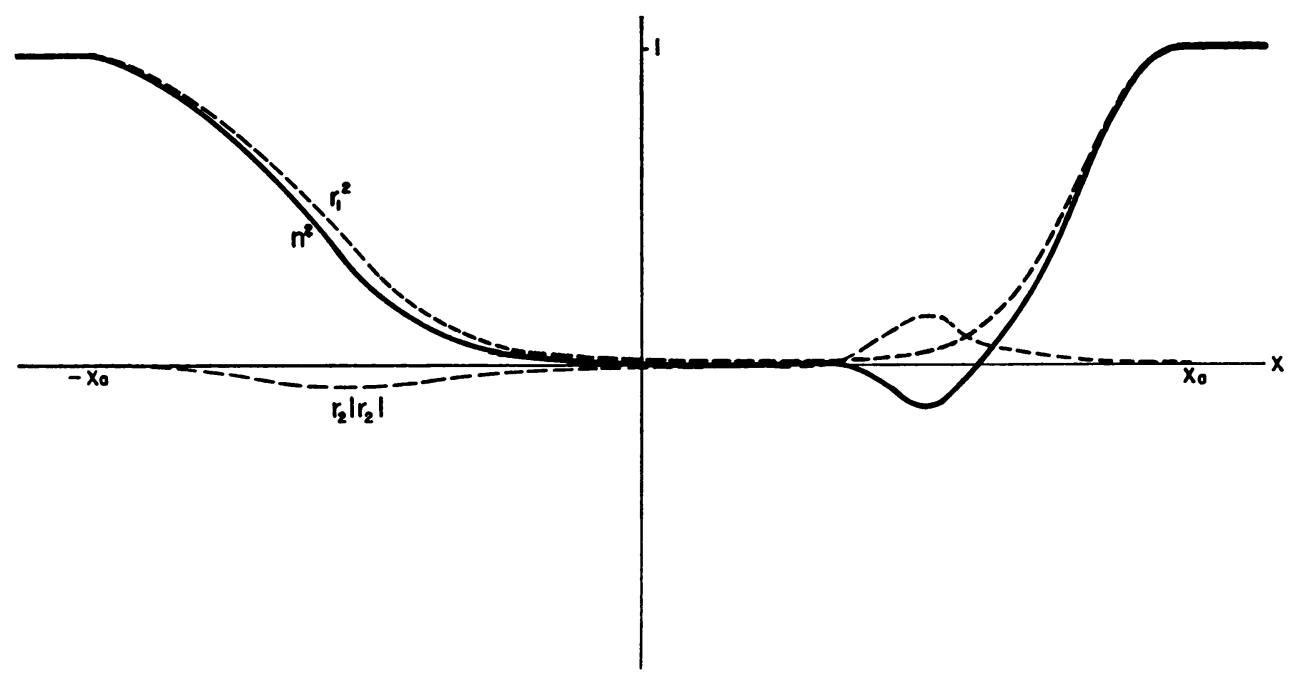

Fig. I6 A NON-REFLECTING MEDIUM (FOR A CERTAIN FREQUENCY). WITH A REGION OF NEGATIVE $n^{2}$. $r_{1}^{2}$ CURVE PRESCRIBED

large) or else one in which $n^{2} \approx 0$ for a long distance (due to $r_{2}$ being positive but small for a long distance, $r_{1}$ growing only slowly). The two cases are illustrated by Figs. 15 and 16. In the case of very thin regions of varying $n^{2}$, in which $r_{1}$ and $r_{2}$ may vary rapidly, these results need not follow (as, for example, Fig. 21, Part IX).

For media which do not satisfy (5), equation (37) and the results derived from it need not hold in cases of non-reflection. This is illustrated in Part IX, Fig. 26.

VIII. Reflection from Media with Two Regions of Negative $\mathrm{n}^{2}$. General media having $n^{2}$ negative in two separated regions cannot be treated by formulae (25) and (27) directly, because of the difficulty of extending $r_{1}, r_{2}, r_{1}^{\prime}$, and $r_{2}^{\prime}$ curves to give values at any one point. Useable formulae for this type of media can be obtained by the following extension of the previous methods: 
Introduce two more solutions of (7)

$$
p^{+}(x)=p_{1}+i p_{2} ; \quad p^{-}(x)=-p_{1}+i p_{2}
$$

where $p_{1}$ and $p_{2}$ are real, such that

$$
p_{1}=|n|, \quad p_{2}=d p_{2} / d x=0 \quad \text { at } \quad x=x_{\mathrm{c}}
$$

where $x_{c}$ is the point at which $n^{2}$ is a maximum $(>0)$ between the two negative regions. Curves of $p_{1}$ and $p_{2}$ may be drawn by the same reasoning as used for $r_{1}$ and $r_{2}$, at least if $\left|n\left(x_{c}\right)\right|$ is not too small. This gives curves such as those shown in Figs. 17 and 18.

By introducing corresponding solutions of (1), and proceeding as before, we get the complex reflection coefficient

$$
R=-\exp \left[2 i k \int_{-x_{a}}^{x_{1}} r_{1} d x\right] \frac{A \exp \left[-2 i k \int_{x_{1}}^{x_{2}} p_{1} d x\right]-B}{C \exp \left[-2 i k \int_{x_{2}}^{x_{2}} p_{1} d x\right]-D}
$$

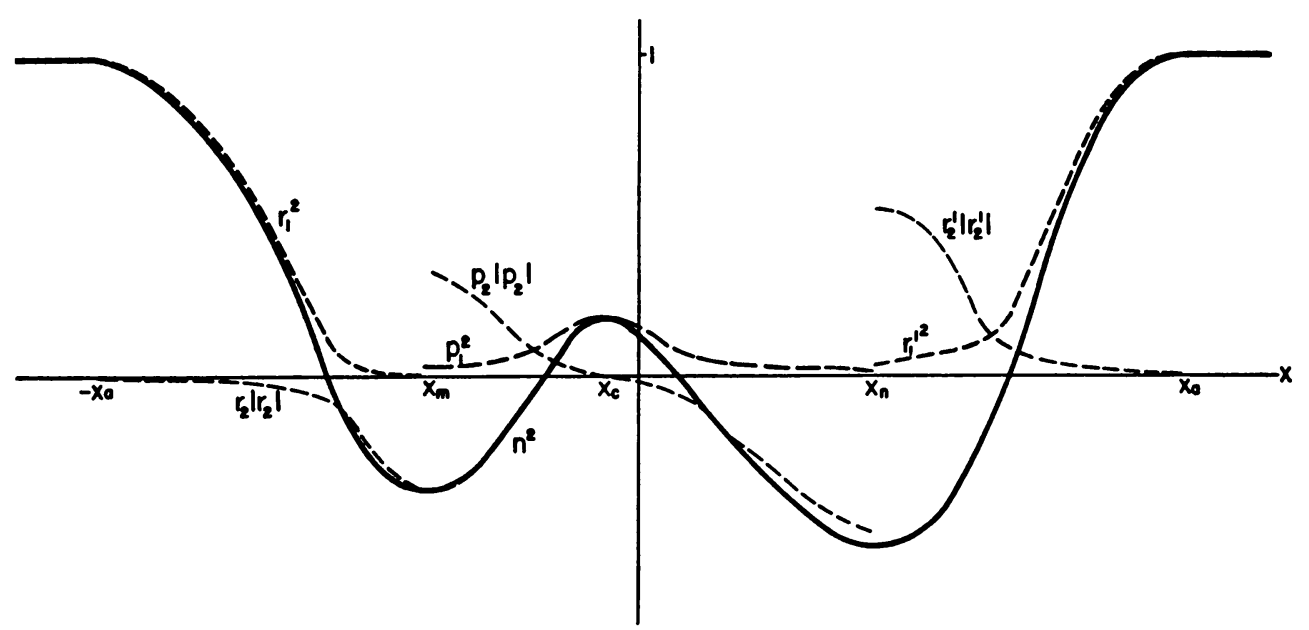

Fig. 17 CURVES FOR A MEDIUM WITH TWO REGIONS OF NEGATIVE $n^{2}$

where

$$
\begin{aligned}
& A=\left(r^{+}-p^{+}\right)_{x=x_{1}}\left(p^{-}-r^{\prime}\right)_{x=x_{2}} \\
& B=\left(r^{+}-p^{-}\right)_{x=x_{1}}\left(p^{+}-r^{\prime}\right)_{x=x_{2}} \\
& C=\left(r^{-}-p^{+}\right)_{x=x_{1}}\left(p^{-}-r^{\prime}\right)_{x=x_{1}} \\
& D=\left(r^{-}-p^{-}\right)_{x=x_{1}}\left(p^{+}-r^{\prime}\right)_{x=x_{2}}
\end{aligned}
$$

and $x_{1}$ and $x_{2}$ are any two values of $x$. For purposes of evaluating (40) we would take $x_{1}$ near $x_{m}$, the point of minimum $n^{2}$ on the left, and $x_{2}$ near $x_{n}$, the point of minimum $n^{2}$ on the right. 


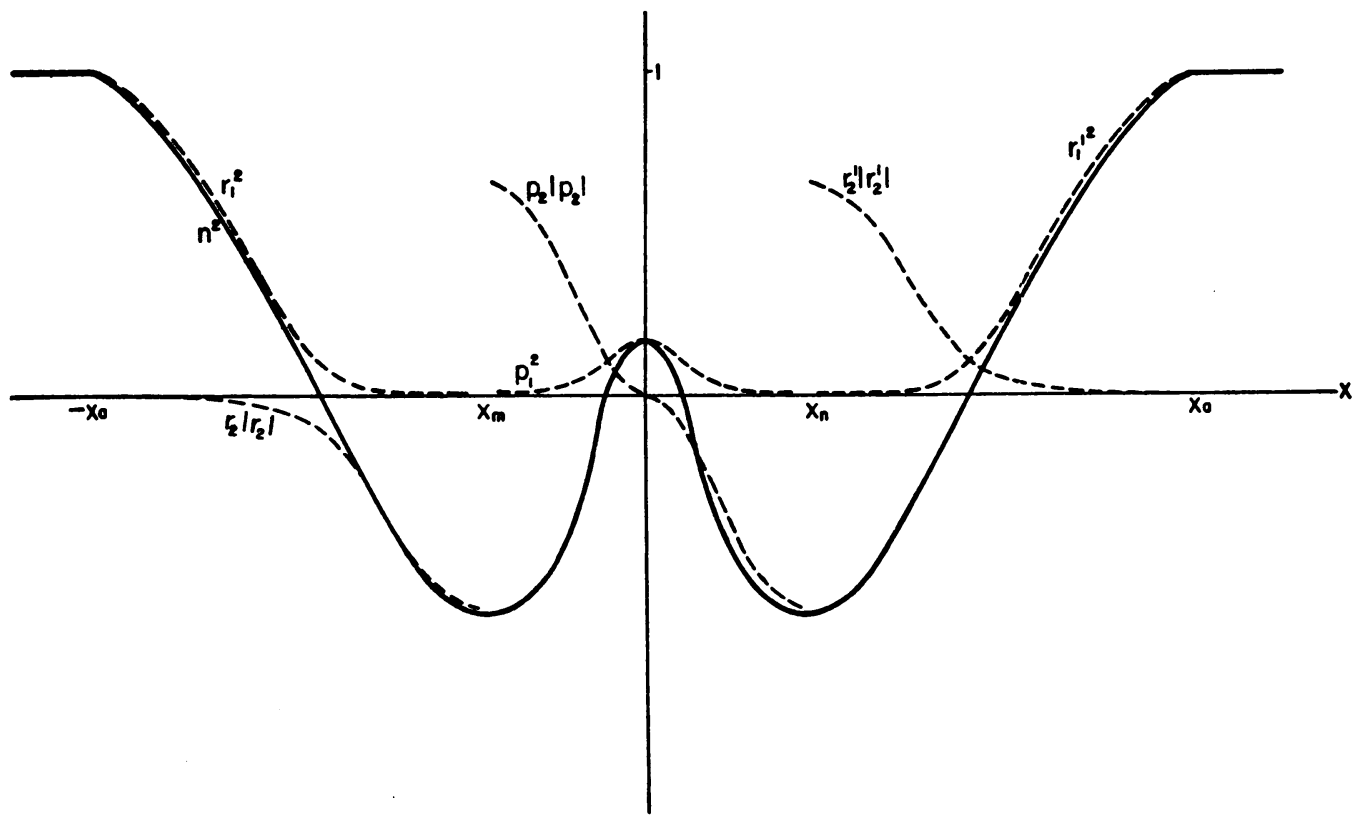

Fig. 18 CURVES FOR A SYMMETRICAL MEDIUM WITH TWO REGIONS OF NEGATIVE $n^{2}$

In slowly varying media, with sufficiently deep minima of $n^{2}$, we will have $-r_{2} \gg r_{1}$, $p_{2} \gg p_{1}$ at $x_{m}$, and $r_{2}^{\prime} \gg r_{1}^{\prime},-p_{2} \gg p_{1}$ at $x_{n}$. Taking $x_{1}=x_{m}, x_{2}=x_{n}$, as we shall from now on, we find $A \approx B \approx C \approx D$ and hence, in most cases, $|R| \approx 1$, the expected result.

However, if two conditions are fulfilled, namely

$$
\begin{gathered}
|A|=|B| \\
\varphi_{A}-2 k \int_{x_{1}}^{x_{2}} p_{1} d x=\varphi_{B}-2 q \pi
\end{gathered}
$$

then we will have, from (40), $|R|=0$. Here $\varphi_{A}=$ phase angle of $A, \varphi_{B}=$ phase angle of $B$, and $q=0, \pm 1, \pm 2, \cdots$.

If the medium is symmetrical, $|A|=|B|$ for all frequencies, as can be shown by choosing $x_{1}=-x_{2}$ and comparing the quantities involved. The second condition, (42), will probably be fulfilled for a whole succession of frequencies. If the medium has curves like those of Fig. 18, where $-r_{2}\left(x_{m}\right) \gg r_{1}\left(x_{m}\right)$ etc., then $\varphi_{A} \approx \varphi_{B} \approx \pi$ nearly independently of frequency. Thus $|R|$ will vanish every time $2 k \int_{x_{1}}^{x_{2}} p_{1} d x$ increases by $2 \pi$ as $k$ varies.

It can further be shown that, for such a medium, the band width $\Delta k$ of passed waves (those having $|R|<1 / \sqrt{2}$ ) is given by

$$
\Delta k \int_{x_{1}}^{x_{2}} p_{1} d x=\left[\frac{4 r_{1} p_{1}}{\left(p_{2}-r_{2}\right)^{2}}\right]_{x=x_{1}} .
$$

On the assumptions made in developing this formula, $\Delta k$ will be a very small quantity. 
These media act as very narrow band pass filters, then. For such a medium, a graph of $|R|$ against $2 k \int_{x_{1}}^{x_{2}} p_{1} d x$ would appear much like that sketched in Fig. 19.

For media having the minimum of $n^{2}$ not very deep, the maxima of $|R|$ would not be so close to 1 , nor would the dips in the curve be as narrow. In a plot of $|R|$ against $k$, some distortion of the graph will occur. This comes from the change of $p_{1}(x)$ with $k$, the changes in $\varphi_{A}$ and $\varphi_{B}$ with $k$, and a possible dispersion $n^{2}=n^{2}(k)$.

Although these results have been derived for symmetrical media, there is no reason to believe that non-symmetrical media need behave much differently. Possibly $|R|$ would not become zero in most cases, even for selected frequencies, but it would probably become small for a sequence of frequencies, with intervening values near 1 . Certainly we can construct non-symmetrical media for which there is no reflection, and in such cases we might expect a marked decrease in $|R|$ for a whole sequence of frequencies.

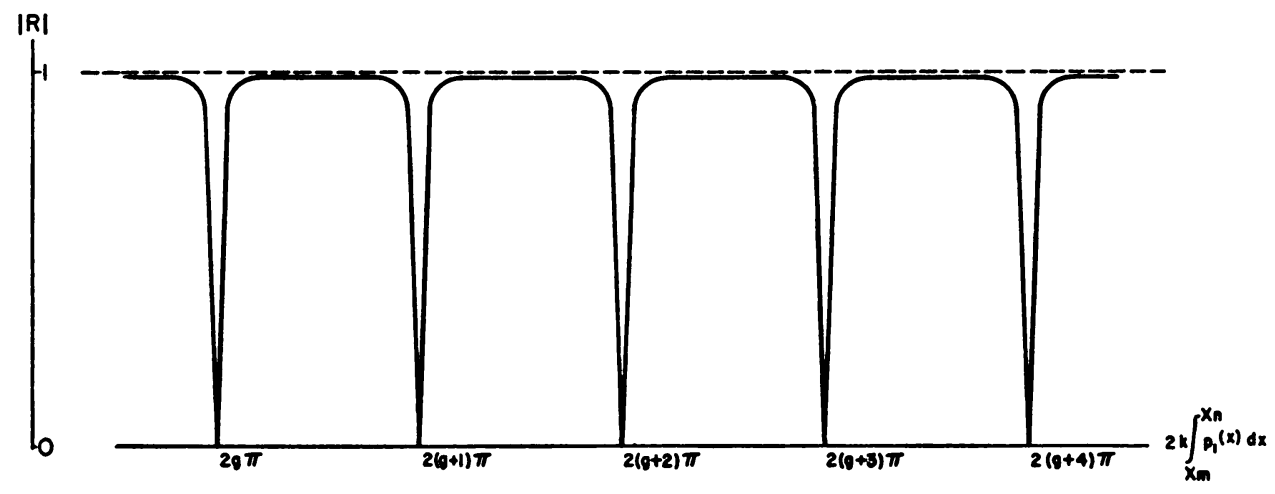

Fig. 19 REFLECTION COEFFICIENT FOR A SYMMETRICAL MEDIUM

The condition (42), and the very nature of media with two regions of negative $n^{2}$, remind one of the theory of the Fabry and Perot interferometer. This suggests as the explanation of perfect transmissions through these media a process of multiple reflection with subsequent interference. It further suggests that the phenomenon will occur to some degree even if the media are somewhat absorbing. Comparison should also be made with the problem of periodically varying media, as treated in Mathieu's equation, where a sequence of bands may be passed. (See Brillouin [15].)

IX. Accurate Reflection Coefficients in Particular Cases. Fig. 20a shows the macroscopic appearance of a symmetrical medium having minimum $n^{2}=0$. This was drawn on the basis of an assigned $r_{1}^{2}$ curve for $|x|>10$, using $k=100$ (i.e. $\lambda_{\text {vac }}=.063$ units). This figure actually shows $n^{2}$ for three media which differ almost inappreciably from one another in the region $|x|<3$ and are identical outside this region. For $|x|<10$, $n^{2}$ is derived by specifying $r_{2}$ (which is continuous, at $x=10$, with the $r_{2}$ curve outside). The three media, with the corresponding $r_{2}$ curves, are shown over the range $-6<$ $x<0$, on a greatly enlarged scale, in Figs. 20b, c, d. They have values of $|R|$ equal to $1 / \sqrt{2}, 0$, and .92 respectively. It is evident from this that very slight changes in the form of $n^{2}$ can change the reflection coefficient remarkably, and that $1 / \sqrt{2}$ is by no means a general result for these media. 


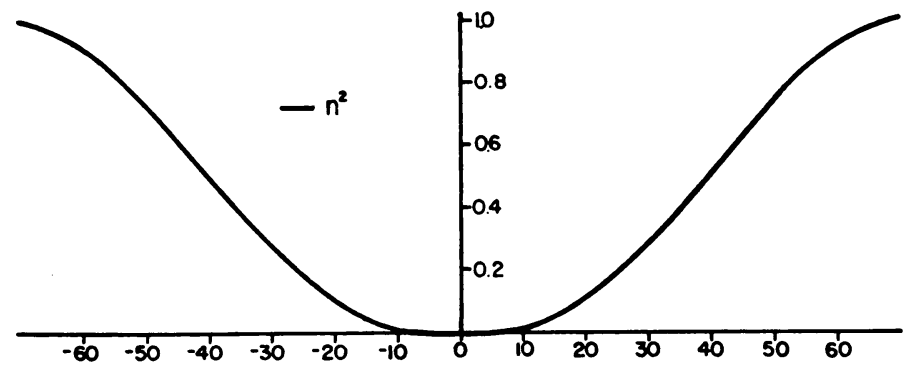

Fig. $20 a$

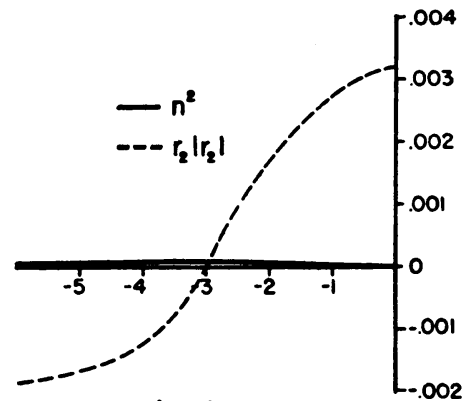

Fig. 20b

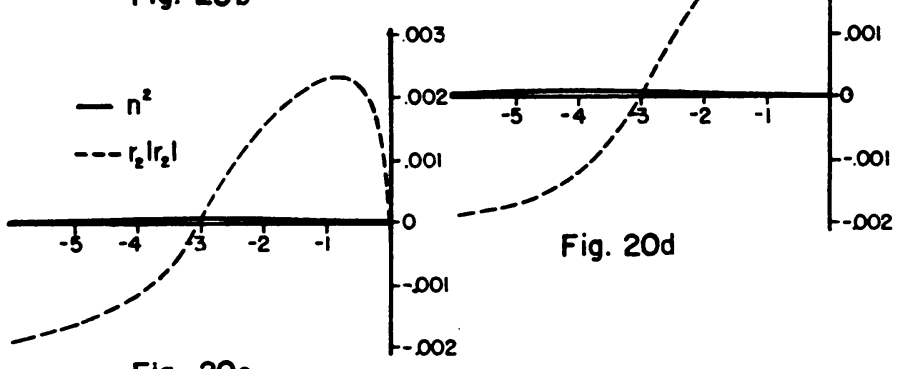

Fig. 20c

Fig. 21 shows a thin, fairly rapidly varying medium, having $n^{2}$ negative in one region only, and having zero reflection for the frequency used in construction. The curve of $r_{2}$ (not shown) was specified, and $k$ taken as 1 ( $\lambda_{\text {vao }}=6.3$ units). In coming from the left, it can be seen that this medium is of Type II and that $D r_{1}$ remains positive

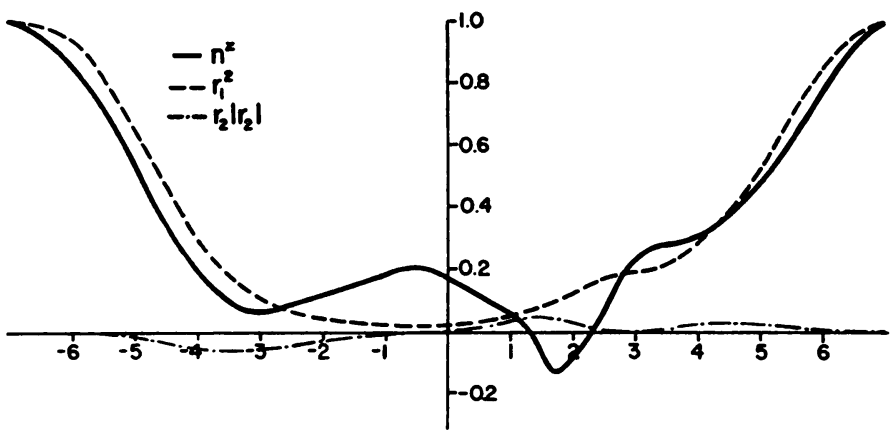

Fig. 21 
throughout the region of negative $n^{2}$. In this case, $\int_{x_{b}}^{x_{d}} r_{1} d x \approx 0.34$ which is less than $\pi$, in agreement with Eq. (31). The reflection coefficient modulus, based on the good path method, is 0.79 , which is in obvious disagreement with the correct value, zero.

Fig. 22 shows, on the right, one half of a symmetrical medium, and on the left, the curves of $r_{1}^{2}$ and $r_{2}\left|r_{2}\right|$ from which it was constructed. Here $k=10, \lambda_{\text {vao }}=0.63$ units. The reflection coefficient for this wavelength has modulus which differs inappreciably from 1 when calculated by either (32) or (33). The transmission coefficient has modulus approximately equal to $\epsilon^{-65}$; the good path method gives $\epsilon^{-69}$.

Fig. 23, shows similar curves for another medium. Here, $k=1$ and $\lambda_{\text {rac }}=6.3$ units for the construction. For these waves, $|R|=0.954$, while the good path method gives $|R|=0.998$. The medium is, of course, rapidly varying and "narrow" relative to the

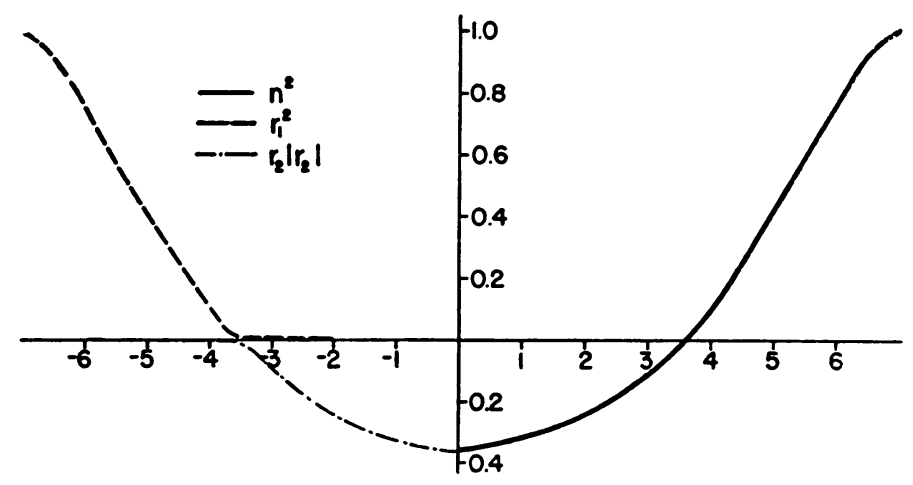

Fig. 22

wavelength used. Figs. 21 and 22 may be compared to see how closely the $r$ curves "stick to" the $n$ curves in the two cases, "fairly slowly" and "fairly rapidly" varying.

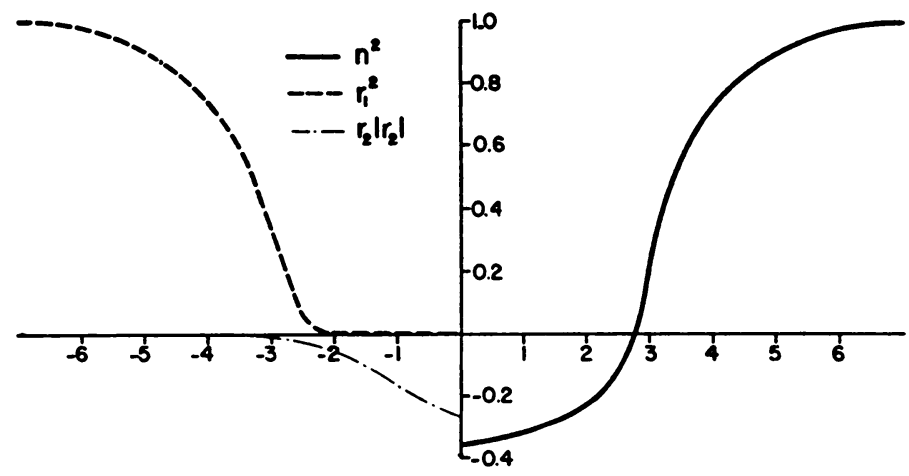

Fig. 23

Figs 24a and b show curves for a medium such as occurs in ionospheric work. The value of $k$ used was 1000 , corresponding to $\lambda_{\text {vas }}=0.0063$ units. For an isonosphere $120 \mathrm{~km}$. thick, this corresponds to $\lambda_{\mathrm{vac}}=63 \mathrm{~m}$, or a frequency of $4.8 \mathrm{mc} / \mathrm{s}$. Reflection is essentially complete here. With such large values of $k$ (for media of this thickness) there is practically no deviation of the $r_{1}^{2}$ and $r_{2}\left|r_{2}\right|$ curves from the limiting forms 
mentioned previously, and almost identical curves would be obtained with any $k$ of this or greater order of magnitude. In the case of the ionosphere, of course, the medium is dispersive and there would be a change of the $n^{2}$ curve as $k$ is changed. Inter-modal coupling must also be included before a complete solution is obtained in this problem.

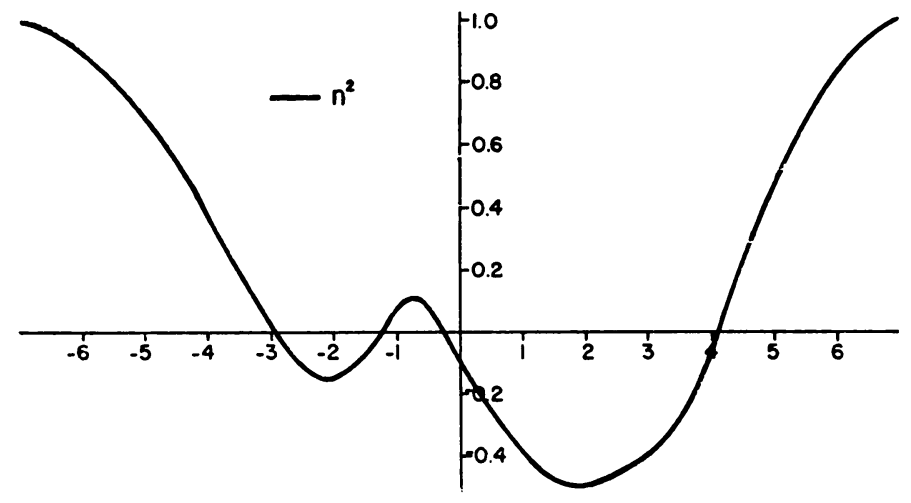

Fig. 240

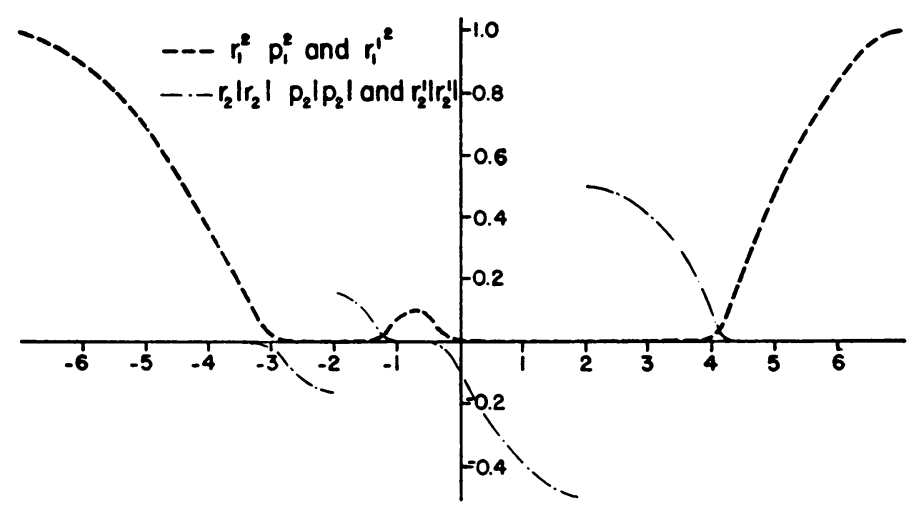

Fiṇ. $24 b$

Fig. 25 shows, on the right, half of a symmetrical medium having two regions of negative $n^{2}$; on the left are curves used in deriving this medium. A value of $k=10$

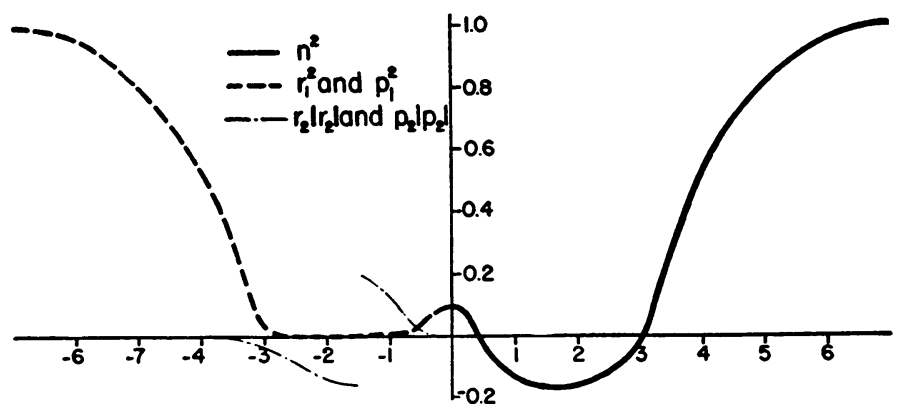

Fig. 25 
was used $\left(\lambda_{\mathrm{va}}=0.63\right.$ units), but similar $r_{1}^{2}$ and $r_{2}\left|r_{2}\right|$ curves would be expected for any $k$ near this. Here $\int_{x_{m}}^{x_{*}} p_{1} d x \approx 0.36$, and at $x_{m}, r_{1} \approx \epsilon^{-1.2 k}, r_{2} \approx 0.4, p_{1} \approx \epsilon^{-0.7 k}$, and $p_{2} \approx 0.4$. These approximations will be fairly good for all $k$ near 10 . Then $\varphi_{\Delta} \approx$ $\varphi_{B} \approx \pi$ as stated before. There will then be no reflection of waves having $k \approx 8.7$, $17.4, \cdots(\approx 2.8 q \pi$ with $q=1,2, \cdots)$. The band widths passed are given by $\Delta k \approx$ $4 \epsilon^{-1.9 k} /(0.8)^{2}(0.36)$. For $k=8.7$ this is $\Delta k \approx 1.1 \times 10^{-6}$ and for $k=17.4$ it is $\Delta k \approx$ $7 \times 10^{-14}$. These are obviously very narrow bands.

Fig. 26 shows a medium which has $n^{2}$ negative only once, and which has $n^{2}=1$ on the left and $n^{2} \approx 0.003$ on the right. There is no reflection for the waves used in construction, which had $k=1$ and $\lambda_{\mathrm{vac}}=6.3$ units.

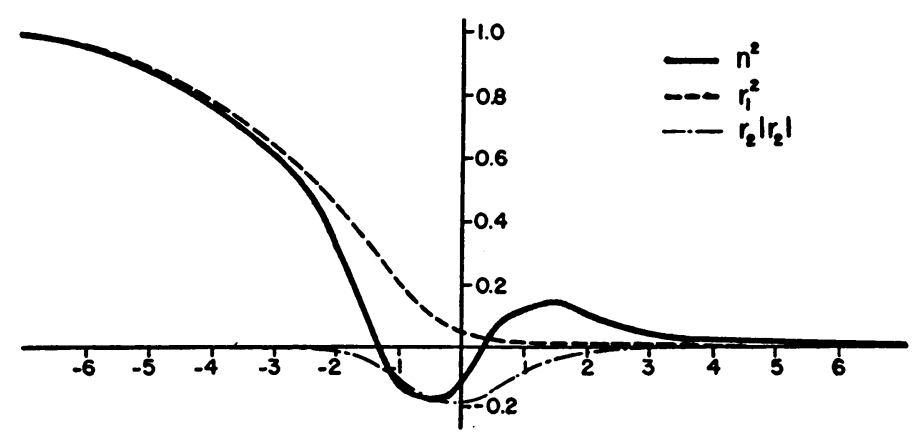

Fig. 26

X. Mass Waves. In quantum theory, an equation of the form of (1) comes from eliminating $t$ from

$$
i \hbar \frac{\partial \Psi}{\partial t}=-\frac{\hbar^{2}}{2 m} \frac{\partial^{2} \Psi}{\partial x^{2}}+V(x) \Psi
$$

where $\hbar$ is Planck's constant divided by $2 \pi, m$ is the mass of a particle, and $V(x)$ is a potential energy field which varies only with the $x$ coordinate. We set

$$
\Psi=f(x) \epsilon^{-i E t / \hbar}
$$

where $E$ is to be the (constant) total energy of the particle. This gives

$$
\frac{d^{2} f}{d x^{2}}+\frac{2 m}{\hbar^{2}}[E-V(x)] f=0 .
$$

The forms of (45) and (4) are identical; the solutions will be identical if we set

$$
k^{2} n^{2}=[E-V(x)] 2 m / \hbar^{2} .
$$

The factorization of the right hand side into $k^{2}$ and $n^{2}$ parts may be done arbitrarily, but the $k^{2}$ part must be independent of $x$ if our previous equations are to hold. If $V=0$ for $|x| \geq x_{a}$, then the most convenient association is

$$
k^{2}=2 m E / \hbar^{2} ; \quad n^{2}=1-V(x) / E
$$

since this gives $n^{2}=1$ for $|x| \geq x_{a}$ as before. The other restrictions previously placed on $n^{2}$ correspond to $V$ being a real continuous function of $x$ which is non-negative between $-x_{a}$ and $x_{a}$. This is then, the potential barrier of quantum mechanics. The cases of maximum $V$ being less than, equal to, or greater than, $E$, correspond to minimum $n^{2}$ being positive, zero, or negative, respectively. 
The results of all the previous parts of the paper may now be applied to the present problem. One modification only is important; we are usually interested in the behaviour of the solutions as $E$ is varied, keeping $m$ and $V$ fixed. This corresponds not only to a variation in $k$ but also to a variation in $n^{2}$-i.e., the media considered are dispersive.

Physically, the results of this paper when applied to mass waves indicate that streams of particles of the proper energy can get through some potential barriers without any reflection. In the case of double barriers, a whole spectrum of such beams could pass. It may be possible to apply this result to obtain sharp ranges of velocities for such experiments as Thomson's e/m determination.

Since the radial wave equation can be put in the same form as (45), the methods of the present paper can be applied in radial problems. For example, exact eigenstate conditions can be established, in terms of $r(x)$, for diatomic molecules. The potentials occurring in such problems do not lead to the type of $n^{2}$ curves considered in this paper, however, so the matter will not be pursued here.

XI. Remarks. The results of this paper are in general agreement with those of other methods. However, by showing that unexpected reflection coefficients may occur, they contain a warning against using the formulae of the other methods in cases where they have not been proven applicable, such as media with two regions of negative $n^{2}$, and media having roots of $n^{2}$ in the complex plane near those on the real $x$-axis (see [15]).

This investigation was carried out at the Radio Physics Laboratory of the Defence Research Board, Ottawa. The problem was proposed by Mr. J. C. W. Scott, whose suggestions and encouragement are much appreciated.

\section{BIBLIOGRAPHY}

[1] Lord Rayleigh, On the propagation of waves through a stratified medium, with special reference to the question of reflection, Proc. Roy. Soc. A 86, 207-226 (1912).

[2] L. Brillouin, La mécanique ondulatoire de Schrödinger; une méthod gênérale de résolution par approximations successives, Comptes Rendus 183, 24-26 (1926).

[3] G. Wentzel, Eine Verallgemeinerung der Quantenbedingungen für die Zwecke der Wellenmechanik, Zeits. f. Physik 38, 518-529 (1926).

[4] H. A. Kramers, Wellenmechanik und halbzahlige Quantisierung, Zeits. f. Physik 39, 828-840 (1926).

[5] E. C. Kemble, $A$ contribution to the theory of the B. W. K. method, Phys. Rev. 48, 549-561 (1935).

[6] E. C. Kemble, The fundamental principles of quantum mechanics, McGraw-Hill Book Company, Inc., New York, 1937, sec. 21.

[7] R. E. Langer, The asymptotic solutions of certain linear ordinary differential equations of the second order, Trans. Amer. Math. Soc. 36, 90-106 (1934).

[8] R. E. Langer, The asymptotic solutions of ordinary linear differential equations of the second order, with special reference to the Stokes phenomenon, Bull. Amer. Math. Soc. 40, 545-582 (1934).

[9] R. E. Langer, On the connection formulas and the solutions of the wave equation, Phys. Rev. 51, 669676 (1937).

10] W. H. Furry, Two notes on phase-integral methods, Phys. Rev. 71, 360-371 (1947).

11] C. Eckart, The penetration of a potential barrier by electrons, Phys. Rev. 35, 1303-1309 (1930).

12] P. S. Epstein, Reflection of waves in an inhomogeneous absorbing medium, Nat. Acad. Sci. 16, 627637 (1930).

13] O. E. Rydbeck, On the propagation of waves in an inhomogeneous medium, Trans. Chalmers U., Gothenburg, Sweden, Nr. 74 (1948).

14] G. Gamow, Zur Quantentheorie des Atomkernes, Zeits. f. Physik 51, 204-212 (1928).

15] L. Brillouin, Wave propagation in periodic structures, McGraw-Hill Book Company, Inc., New York, 1946, chap. VIII.

16] S. A. Schelkunoff, Remarks concerning wave propagation in stratified media, Communications on Pure and Applied Mathematics 4, 117-128 (1951). 